PROCEEDINGS OF THE

AMERICAN MATHEMATICAL SOCIETY

Volume 136, Number 7, July 2008, Pages 2375-2386

S 0002-9939(08)09239-3

Article electronically published on March 19, 2008

\title{
STABLE RANK OF LEAVITT PATH ALGEBRAS
}

\author{
P. ARA AND E. PARDO
}

(Communicated by Birge Huisgen-Zimmerman)

\begin{abstract}
We characterize the values of the stable rank for Leavitt path algebras by giving concrete criteria in terms of properties of the underlying graph.
\end{abstract}

\section{INTRODUCTION AND BACKGROUND}

Leavitt path algebras have been recently introduced in [1] and 5. Given an arbitrary (but fixed) field $K$ and a row-finite graph $E$, the Leavitt path algebra $L_{K}(E)$ is the algebraic analogue of the Cuntz-Krieger algebra $C^{*}(E)$ described in [11. Several interesting ring-theoretic properties have been characterized for this class of algebras. For instance, the Leavitt path algebras which are purely infinite simple have been characterized in [2], and 7] contains a characterization of the Leavitt path algebras which are exchange rings in terms of condition $(\mathrm{K})$, a purely graph-theoretic condition defined below.

In this paper, we show that the only possible values of the (Bass) stable rank of a Leavitt path algebra are 1, 2 and $\infty$. Moreover a precise characterization in terms of properties of the graph of the value of the stable rank is provided (Theorem 2.8). A similar result was obtained in [7, Theorem 7.6] under the additional hypothesis that the graph satisfies condition (K) (equivalently, $L(E)$ is an exchange ring). Many tools of the proof of that result must be reworked in our general situation. We have obtained in several situations simpler arguments that work without the additional hypothesis of condition $(\mathrm{K})$ on the graph. Another new feature of our approach is a detailed analysis of the stable rank in extensions of Leavitt path algebras of stable rank 2, in order to show that the stable rank of these extensions cannot shift to 3 . Our main tool for this study is the well-known concept of elementary rank; see for example [10, Chapter 11].

Received by the editors February 2, 2007, and, in revised form, April 16, 2007, and May 22, 2007

2000 Mathematics Subject Classification. Primary 16D70.

Key words and phrases. Leavitt path algebra, stable rank.

The first author was partially supported by the DGI and European Regional Development Fund jointly through Project MTM2005-00934.

The second author was partially supported by the DGI and European Regional Development Fund jointly through Project MTM2004-00149, and by PAI III grants FQM-298 and P06-FQM1889 of the Junta de Andalucía.

Both authors are partially supported by the Comissionat per Universitats i Recerca de la Generalitat de Catalunya.

(C)2008 American Mathematical Society Reverts to public domain 28 years from publication 
The (topological) stable rank of the Cuntz-Krieger algebras $C^{*}(E)$ was computed in [8]. This paper has been the inspiration for the general strategy of the proof here. Note that, by a result of Herman and Vaserstein [9], the topological and the Bass stable ranks coincide for $C^{*}$-algebras. For the sake of comparison, let us mention that, although the possible values of the stable rank of $C^{*}(E)$ are also 1,2 and $\infty$, it turns out that there are graphs $E$ such that the stable rank of $C^{*}(E)$ is 1 while the stable rank of $L(E)$ is 2. Concretely, if $E$ is a graph such that no cycle has an exit and $E$ contains some cycle, then the stable rank of $C^{*}(E)$ is 1 by 8 , Theorem 3.4 , but the stable rank of $L(E)$ is 2 by Theorem 2.8,

In this paper, we describe the Leavitt path algebras following the presentation of [5. Sections 3 and 5], but using the notation of [1] for the elements.

A (directed) graph $E=\left(E^{0}, E^{1}, r, s\right)$ consists of two countable sets $E^{0}, E^{1}$ and maps $r, s: E^{1} \rightarrow E^{0}$. The elements of $E^{0}$ are called vertices and the elements of $E^{1}$ edges.

A vertex which emits no edges is called a sink. A graph $E$ is finite if $E^{0}$ and $E^{1}$ are finite sets. If $s^{-1}(v)$ is a finite set for every $v \in E^{0}$, then the graph is called row-finite. A path $\mu$ in a graph $E$ is a sequence of edges $\mu=\left(\mu_{1}, \ldots, \mu_{n}\right)$ such that $r\left(\mu_{i}\right)=s\left(\mu_{i+1}\right)$ for $i=1, \ldots, n-1$. In such a case, $s(\mu):=s\left(\mu_{1}\right)$ is the source of $\mu$ and $r(\mu):=r\left(\mu_{n}\right)$ is the range of $\mu$. If $s(\mu)=r(\mu)$ and $s\left(\mu_{i}\right) \neq s\left(\mu_{j}\right)$ for every $i \neq j$, then $\mu$ is called a cycle. We say that a cycle $\mu=\left(\mu_{1}, \ldots, \mu_{n}\right)$ has an exit if there is a vertex $v=s\left(\mu_{i}\right)$ and an edge $f \in s^{-1}(v) \backslash\left\{\mu_{i}\right\}$. If $v=s(\mu)=r(\mu)$ and $s\left(\mu_{i}\right) \neq v$ for every $i>1$, then $\mu$ is a called a closed simple path based at $v$. We denote by $C S P_{E}(v)$ the set of closed simple paths in $E$ based at $v$. For a path $\mu$ we denote by $\mu^{0}$ the set of its vertices, i.e., $\left\{s\left(\mu_{1}\right), r\left(\mu_{i}\right) \mid i=1, \ldots, n\right\}$. For $n \geq 2$ we define $E^{n}$ to be the set of paths of length $n$, and $E^{*}=\bigcup_{n \geq 0} E^{n}$ the set of all paths. We define a relation $\geq$ on $E^{0}$ by setting $v \geq w$ if there is a path $\mu \in E^{*}$ with $s(\mu)=v$ and $r(\mu)=w$. A subset $H$ of $E^{0}$ is called hereditary if $v \geq w$ and $v \in H$ imply $w \in H$. A set is saturated if every vertex which feeds into $H$ and only into $H$ is again in $H$, that is, if $s^{-1}(v) \neq \emptyset$ and $r\left(s^{-1}(v)\right) \subseteq H$ imply $v \in H$. Denote by $\mathcal{H}$ (or by $\mathcal{H}_{E}$ when it is necessary to emphasize the dependence on $E$ ) the set of hereditary saturated subsets of $E^{0}$. We denote by $E^{\infty}$ the set of infinite paths $\gamma=\left(\gamma_{n}\right)_{n=1}^{\infty}$ of the graph $E$ and by $E^{\leq \infty}$ the set $E^{\infty}$ together with the set of finite paths in $E$ whose end vertex is a sink. We say that a vertex $v$ in a graph $E$ is cofinal if for every $\gamma \in E \leq \infty$ there is a vertex $w$ in the path $\gamma$ such that $v \geq w$. We say that a graph $E$ is cofinal if so are all the vertices of $E$. According to 7 , Lemma 2.8], this is equivalent to the fact that $\mathcal{H}=\left\{\emptyset, E^{0}\right\}$.

Let $E=\left(E^{0}, E^{1}, r, s\right)$ be a graph, and let $K$ be a field. We define the Leavitt path algebra $L_{K}(E)$ associated with $E$ as the $K$-algebra generated by a set $\left\{v \mid v \in E^{0}\right\}$ of pairwise orthogonal idempotents, together with a set of variables $\left\{e, e^{*} \mid e \in E^{1}\right\}$, which satisfy the following relations:

(1) $s(e) e=e r(e)=e$ for all $e \in E^{1}$.

(2) $r(e) e^{*}=e^{*} s(e)=e^{*}$ for all $e \in E^{1}$.

(3) $e^{*} e^{\prime}=\delta_{e, e^{\prime}} r(e)$ for all $e, e^{\prime} \in E^{1}$.

(4) $v=\sum_{\left\{e \in E^{1} \mid s(e)=v\right\}} e e^{*}$ for every $v \in E^{0}$ that emits edges.

Note that the relations above imply that $\left\{e e^{*} \mid e \in E^{1}\right\}$ is a set of pairwise orthogonal idempotents in $L_{K}(E)$. Note also that if $E$ is a finite graph, then we have $\sum_{v \in E^{0}} v=1$. In general the algebra $L_{K}(E)$ is not unital, but it can be written as a direct limit of unital Leavitt path algebras (with nonunital transition maps), 
so that it is an algebra with local units (recall that a local unit in a ring $R$ is an increasing net of idempotents $\left\{e_{\lambda}\right\}_{\lambda \in \Lambda} \subset R$ such that for each $a \in R$ there exists $\mu \in \Lambda$ with $\left.a=a e_{\mu}=e_{\mu} a\right)$. In this paper, we will be concerned only with row-finite graphs $E$, and we will work with Leavitt path algebras over an arbitrary but fixed field $K$. We will usually suppress the field from the notation.

Recall that $L(E)$ has a $\mathbb{Z}$-grading. For every $e \in E^{1}$, set the degree of $e$ as 1 , the degree of $e^{*}$ as -1 , and the degree of every element in $E^{0}$ as 0 . Then we obtain a well-defined degree on the Leavitt path $K$-algebra $L(E)$; thus, $L(E)$ is a $\mathbb{Z}$-graded algebra:

$$
L(E)=\bigoplus_{n \in \mathbb{Z}} L(E)_{n}, \quad L(E)_{n} L(E)_{m} \subseteq L(E)_{n+m}, \text { for all } n, m \in \mathbb{Z} .
$$

An ideal $I$ of a $\mathbb{Z}$-graded algebra $A=\bigoplus_{n \in \mathbb{Z}} A_{n}$ is a graded ideal in case $I=$ $\bigoplus_{n \in \mathbb{Z}}\left(I \cap A_{n}\right)$. By [5, Proposition 5.2 and Theorem 5.3], an ideal $J$ of $L(E)$ is graded if and only if it is generated by idempotents; in fact, $J$ is a graded ideal if and only if $J$ coincides with the ideal $I(H)$ of $L(E)$ generated by $H$, where $H=J \cap E^{0} \in \mathcal{H}_{E}$. Indeed, the map $H \mapsto I(H)$ defines a lattice isomorphism between $\mathcal{H}_{E}$ and $\mathcal{L}_{\text {gr }}(L(E))$.

Recall that a graph $E$ satisfies condition (L) if every closed simple path has an exit, and satisfies condition $(\mathrm{K})$ if for each vertex $v$ on a closed simple path there exist at least two distinct closed simple paths $\alpha, \beta$ based at $v$.

Section 1 contains some basic information on the structure of Leavitt path algebras, which will be very useful for the computations in Section 2 of the stable rank of such algebras. Finally, Section 3 contains some illustrative examples of Leavitt path algebras.

\section{BASIC FACTS}

For a graph $E$ and a hereditary subset $H$ of $E^{0}$, we denote by $E_{H}$ the restriction graph

$$
\left(H,\left\{e \in E^{1} \mid s(e) \in H\right\},\left.r\right|_{\left(E_{H}\right)^{1}},\left.s\right|_{\left(E_{H}\right)^{1}}\right) .
$$

Observe that if $H$ is finite, then $L\left(E_{H}\right)=p_{H} L(E) p_{H}$, where $p_{H}=\sum_{v \in H} v \in L(E)$. On the other hand, for $X \in \mathcal{H}_{E}$, we denote by $E / X$ the quotient graph

$$
\left(E^{0} \backslash X,\left\{e \in E^{1} \mid r(e) \notin X\right\},\left.r\right|_{(E / X)^{1}},\left.s\right|_{(E / X)^{1}}\right) .
$$

By [7, Lemma 2.3(i)] we have a natural isomorphism $L(E) / I(X) \cong L(E / X)$ for $X \in \mathcal{H}_{E}$. Our next result shows that $I(X)$ is also a Leavitt path algebra.

Definition 1.1 ([8, Definition 1.3]). Let $E$ be a graph, and let $\emptyset \neq X \in \mathcal{H}_{E}$. Define

$$
\begin{array}{r}
F_{E}(X)=\left\{\alpha=\left(\alpha_{1}, \ldots, \alpha_{n}\right) \in E^{n} \mid n \geq 1, s\left(\alpha_{1}\right) \in E^{0} \backslash X, r\left(\alpha_{i}\right) \in E^{0} \backslash X\right. \\
\\
\left.\quad \text { for every } i<n, r\left(\alpha_{n}\right) \in X\right\} .
\end{array}
$$

Let $\overline{F_{E}(X)}=\left\{\bar{\alpha} \mid \alpha \in F_{E}(X)\right\}$. Then, we define the graph ${ }_{X} E=\left({ }_{X} E^{0},{ }_{X} E^{1}, s^{\prime}, r^{\prime}\right)$ as follows:

(1) ${ }_{X} E^{0}=X \cup F_{E}(X)$.

(2) ${ }_{X} E^{1}=\left\{e \in E^{1} \mid s(e) \in X\right\} \cup \overline{F_{E}(X)}$.

(3) For every $e \in E^{1}$ with $s(e) \in X, s^{\prime}(e)=s(e)$ and $r^{\prime}(e)=r(e)$.

(4) For every $\bar{\alpha} \in \overline{F_{E}(X)}, s^{\prime}(\bar{\alpha})=\alpha$ and $r^{\prime}(\bar{\alpha})=r(\alpha)$. 
Lemma 1.2. Let $E$ be a graph, and let $\emptyset \neq X \in \mathcal{H}_{E}$. Then, $I(X) \cong L\left({ }_{X} E\right)$ (as nonunital rings).

Proof. We define a map $\phi: L\left({ }_{X} E\right) \rightarrow I(X)$ by the following rule: (i) for every $v \in X, \phi(v)=v$; (ii) for every $\alpha \in F_{E}(X), \phi(\alpha)=\alpha \alpha^{*}$; (iii) for every $e \in$ $E^{1}$ with $s(e) \in X, \phi(e)=e$ and $\phi\left(e^{*}\right)=e^{*}$; (iv) for every $\bar{\alpha} \in \overline{F_{E}(X)}, \phi(\bar{\alpha})=\alpha$ and $\phi\left(\bar{\alpha}^{*}\right)=\alpha^{*}$.

By definition, it is clear that the images of the generators of $L\left({ }_{X} E\right)$ satisfy the relations defining $L\left({ }_{X} E\right)$. Thus, $\phi$ is a well-defined $K$-algebra morphism.

To see that $\phi$ is onto, it is enough to show that every vertex of $X$ and every finite path $\alpha$ of $E$ which ranges in $X$ are in the image of $\phi$. For any $v \in X, \phi(v)=v$, so that this case is clear. Now, let $\alpha=\left(\alpha_{1}, \ldots, \alpha_{n}\right)$ with $\alpha_{i} \in E^{1}$. If $s\left(\alpha_{1}\right) \in X$, then $\alpha=\phi\left(\alpha_{1}\right) \cdots \phi\left(\alpha_{n}\right)$. Suppose that $s\left(\alpha_{1}\right) \in E^{0} \backslash X$ and $r\left(\alpha_{n}\right) \in X$. Then, there exists $1 \leq j \leq n-1$ such that $r\left(\alpha_{j}\right) \in E^{0} \backslash X$ and $r\left(\alpha_{j+1}\right) \in X$. Thus, $\alpha=\left(\alpha_{1}, \ldots, \alpha_{j+1}\right)\left(\alpha_{j+2}, \ldots, \alpha_{n}\right)$, where $\beta=\left(\alpha_{1}, \ldots, \alpha_{j+1}\right) \in F_{E}(X)$. Hence, $\alpha=\phi(\bar{\beta}) \phi\left(\alpha_{j+2}\right) \cdots \phi\left(\alpha_{n}\right)$.

To show injectivity, notice that, for every $\alpha \in F_{E}(X), \alpha=\overline{\alpha \alpha}^{*}$. Hence, every element $t \in L\left({ }_{X} E\right)$ can be written as

$$
t=\sum_{\alpha, \beta \in F_{E}(X)} \bar{\alpha} a_{\alpha, \beta} \bar{\beta}^{*}
$$

where $a_{\alpha, \beta} \in L\left(E_{X}\right)$. Suppose that $0 \neq \operatorname{Ker}(\phi)$, and let $0 \neq t \in \operatorname{Ker}(\phi)$ be written as in (11). By definition of the map $\phi$,

$$
0=\phi(t)=\sum_{\alpha, \beta \in F_{E}(X)} \alpha a_{\alpha, \beta} \beta^{*} .
$$

Let $\alpha_{0} \in F_{E}(X)$ with maximal length among those appearing (with a nonzero coefficient) in the expression (2). Then, for any other $\alpha \in F_{E}(X)$ appearing in the same expression, $\alpha_{0}^{*} \cdot \alpha$ is 0 if $\alpha \neq \alpha_{0}$ or $r\left(\alpha_{0}\right)$ if $\alpha=\alpha_{0}$. Thus,

$$
0=\sum_{\alpha, \beta \in F_{E}(X)} \alpha_{0}^{*} \alpha a_{\alpha, \beta} \beta^{*}=\sum_{\beta \in F_{E}(X)} a_{\alpha_{0}, \beta} \beta^{*} .
$$

Now, let $\beta_{0} \in F_{E}(X)$ with maximal length among those appearing in the expression (3). The same argument as above shows that

$$
0=\sum_{\beta \in F_{E}(X)} a_{\alpha_{0}, \beta} \beta^{*} \beta_{0}=a_{\alpha_{0}, \beta_{0}} .
$$

But $0 \neq a_{\alpha_{0}, \beta_{0}}$ by hypothesis, and we reach a contradiction. Thus, we conclude that $\phi$ is injective.

Lemma 1.3. Let $R$ be a ring, and let $I \triangleleft R$ an ideal with local unit. If there exists an ideal $J \triangleleft I$ such that $I / J$ is a unital simple ring, then there exists an ideal $M \triangleleft R$ such that $R / M \cong I / J$.

Proof. Given $a \in J$, there exists $x \in I$ such that $a=a x=x a$. Thus, $J \subseteq J I$, and $J \subseteq I J$. Hence, $J \triangleleft R$.

By hypothesis, there exists an element $e \in I$ such that $\bar{e} \in I / J$ is the unit. Consider the set $\mathcal{C}$ of ideals $L$ of $R$ such that $J \subseteq L$ and $e \notin L$. If we order $\mathcal{C}$ by inclusion, it is easy to see that it is inductive. Thus, by Zorn's Lemma, there exists 
a maximal element of $\mathcal{C}$, say $M$. Then, $J \subseteq M \cap I \varsubsetneqq I$, whence $J=M \cap I$ by the maximality of $J$ in $I$. Thus,

$$
I / J=I /(M \cap I) \cong I+M / M \triangleleft R / M .
$$

Suppose that $R \neq I+M$. Clearly, $\bar{e} \in I+M / M$ is a unit. Thus, $\bar{e}$ is a central idempotent of $R / M$ generating $I+M / M$. So, $L=\{a-a \bar{e} \mid a \in R / M\}$ is an ideal of $R / M$, while

$$
R / M=\bar{e}(R / M)+L
$$

the sum being an internal direct sum. If $\pi: R \rightarrow R / M$ is the natural projection map, then $\pi^{-1}(L)=M+\{a-a e \mid a \in R\}$ is an ideal of $R$ containing $M$ (and so $J)$. If $e \in \pi^{-1}(L)$, then $L=R / M$, which is impossible. Hence, $\pi^{-1}(L) \in \mathcal{C}$, and contains strictly $M$, contradicting the maximality of $M$ in $\mathcal{C}$. Thus, $I+M=R$, and so $R / M \cong I / J$, as desired.

Corollary 1.4. Let $E$ be a graph, and let $H \in \mathcal{H}_{E}$. If there exists $J \triangleleft I(H)$ such that $I(H) / J$ is a unital simple ring, then there exists an ideal $M \triangleleft L(E)$ such that $L(E) / M \cong I(H) / J$.

Proof. By Lemma 1.2, $I(H) \cong L\left({ }_{H} E\right)$, whence $I(H)$ has a local unit. Thus, the result holds by Lemma 1.3 .

Recall that an idempotent $e$ in a ring $R$ is called infinite if $e R$ is isomorphic as a right $R$-module to a proper direct summand of itself. A simple ring $R$ is called purely infinite in case every nonzero right ideal of $R$ contains an infinite idempotent. See [4 for some basic properties of purely infinite simple rings and [2, Theorem 11] for a characterization of purely infinite simple Leavitt path algebras in terms of properties of the graph.

Proposition 1.5. Let $E$ be a row-finite graph, and let $J$ be a maximal two-sided ideal of $L(E)$. If $L(E) / J$ is a unital purely infinite simple ring, then $J \in \mathcal{L}_{g r}(L(E))$.

Proof. Let $a$ be an element of $L(E)$ such that $a+J$ is the unit in $L(E) / J$. There are $v_{1}, \ldots, v_{n} \in E^{0}$ such that $a \in p L(E) p$, where $p=v_{1}+\cdots+v_{n} \in L(E)$. Since $a v=v a=0$ for all $v \in E^{0} \backslash\left\{v_{1}, \ldots, v_{n}\right\}$, it follows that the hereditary saturated set $X=\left\{v \in E^{0} \mid v \in J\right\}$ is cofinite in $E^{0}$ and thus passing to $L(E) / I(X) \cong L(E / X)$, we can assume that $E$ is a finite graph and that $E^{0} \cap J=\emptyset$.

Since $E$ is finite, the lattice $\mathcal{L}_{\mathrm{gr}}(L(E))$ of graded ideals (equivalently, idempotentgenerated ideals) of $L(E)$ is finite by [5, Theorem 5.3], so that there exists a nonempty $H \in \mathcal{H}_{E}$ such that $I=I(H)$ is minimal as a graded ideal. Since $I+J=L(E)$ by our assumption that $J \cap E^{0}=\emptyset$, we have

$$
I /(I \cap J) \cong L(E) / J
$$

so that $I$ has a unital purely infinite simple quotient. Since $I \cong L\left({ }_{H} E\right)$ and $J \cap I$ does not contain nonzero idempotents, it follows from our previous argument that ${ }_{H} E$ is finite and so $I$ is unital. So $I=e L(E)$ for a central idempotent $e$ in $L(E)$. Since $I$ is graded-simple, [5, Remark 6.7] and [2, Theorem 11] imply that $I$ is either $M_{n}(K)$ or $M_{n}\left(K\left[x, x^{-1}\right]\right)$ for some $n \geq 1$ or it is simple purely infinite. Since $I$ has a quotient algebra which is simple purely infinite, it follows that $I \cap J=\emptyset$ and $J=(1-e) L(E)$ is a graded ideal. Indeed we get $e=1$ because we are assuming that $J$ does not contain nonzero idempotents. 
Notice that, as a consequence of Proposition [1.5] and [2, Theorem 11], we get the following generalization of [7, Lemma 7.2], which is analogous to [8, Proposition 3.1].

Lemma 1.6. Let $E$ be a row-finite graph. Then, $L(E)$ has a unital purely infinite simple quotient if and only if there exists $H \in \mathcal{H}_{E}$ such that the quotient graph $E / H$ is nonempty, finite, cofinal, contains no sinks and each cycle has an exit.

\section{Stable RANK For Leavitt Path algebras}

Let $S$ be any unital ring containing an associative ring $R$ as a two-sided ideal. The following definitions can be found in [13. A column vector $b=\left(b_{i}\right)_{i=1}^{n}$ is called $R$-unimodular if $b_{1}-1, b_{i} \in R$ for $i>1$ and there exist $a_{1}-1, a_{i} \in R(i>1)$ such that $\sum_{i=1}^{n} a_{i} b_{i}=1$. The stable rank of $R$ (denoted by $\operatorname{sr}(R)$ ) is the least natural number $m$ for which for any $R$-unimodular vector $b=\left(b_{i}\right)_{i=1}^{m+1}$ there exist $v_{i} \in R$ such that the vector $\left(b_{i}+v_{i} b_{m+1}\right)_{i=1}^{m}$ is $R$-unimodular. If such a natural $m$ does not exist, we say that the stable rank of $R$ is infinite.

Lemma 2.1 (cf. [7, Lemma 7.1]). Let $E$ be an acyclic graph. Then, the stable rank of $L(E)$ is 1 .

Lemma 2.2. Let $E$ be a graph. If there exists a unital purely infinite simple quotient of $L(E)$, then the stable rank of $L(E)$ is $\infty$.

Proof. If there exists a maximal ideal $M \triangleleft L(E)$ such that $L(E) / M$ is a unital purely infinite simple ring, then $\operatorname{sr}(L(E) / M)=\infty$ (see e.g. [4]). Since $\operatorname{sr}(L(E) / M) \leq$ $\operatorname{sr}(L(E))$ (see [13, Theorem 4]), we conclude that $\operatorname{sr}(L(E))=\infty$.

We adapt the following terminology from $[8$ : we say that a graph $E$ has isolated cycles if whenever $\left(a_{1}, \ldots, a_{n}\right)$ and $\left(b_{1}, \ldots, b_{m}\right)$ are closed simple paths such that $s\left(a_{i}\right)=s\left(b_{j}\right)$ for some $i, j$, then $a_{i}=b_{j}$. Notice that, in particular, if $E$ has isolated cycles, the only closed simple paths it can contain are cycles.

Lemma 2.3 (cf. 8, Lemma 3.2], 7, Lemma 7.4]). Let $E$ be a graph. If $L(E)$ does not have any unital purely infinite simple quotient, then there exists a graded ideal $J \triangleleft L(E)$ with $\operatorname{sr}(J) \leq 2$ such that $L(E) / J$ is isomorphic to the Leavitt path algebra of a graph with isolated cycles. Moreover $\operatorname{sr}(J)=1$ if and only if $J=0$.

Proof. Set

$$
X_{0}=\left\{v \in E^{0} \mid \exists e \neq f \in E^{1} \text { with } s(e)=s(f)=v, r(e) \geq v, r(f) \geq v\right\},
$$

and let $X$ be the hereditary saturated closure of $X_{0}$. Consider $J=I(X)$. Then $J$ is a graded ideal of $L(E)$ and $L(E) / J \cong L(E / X)$ by [7. Lemma 2.3(1)]. It is clear from the definition of $X_{0}$ that $E / X$ is a graph with isolated cycles.

It remains to show that $\operatorname{sr}(J) \leq 2$ and that $\operatorname{sr}(J)=2$ if $J \neq 0$. The proof of these facts follows the lines of the proof of [7, Lemma 7.4], using Corollary 1.4 instead of [7. Proposition 5.4] and Lemma 1.2 instead of [7, Lemma 5.2].

Definition 2.4. Let $A$ be a unital ring with stable rank $n$. We say that $A$ has stable rank closed by extensions in case for any unital ring extension

$$
0 \longrightarrow I \longrightarrow B \longrightarrow 0
$$

of $A$ with $\operatorname{sr}(I) \leq n$ we have $\operatorname{sr}(B)=n$. 
Recall that a unital ring $R$ has elementary rank $n$, denoted by $\operatorname{er}(R)=n$, in case that, for every $t \geq n+1$, the elementary group $E_{t}(R)$ acts transitively on the set $U_{c}(t, R)$ of $t$-unimodular columns with coefficients in $R$; see [10, 11.3.9].

In the next lemma, we collect some properties that we will need in the sequel.

Lemma 2.5. Let $A$ be a unital ring. Assume that $\operatorname{sr}(A)=n<\infty$.

(1) If $\operatorname{er}(A)<n$, then $M_{m}(A)$ has stable rank closed by extensions for every $m \geq 1$.

(2) Let $D$ be any (commutative) Euclidean domain such that $\operatorname{sr}(D)>1$ and let $m$ be a positive integer. Then $\operatorname{sr}\left(M_{m}(D)\right)=2$ and $\operatorname{er}\left(M_{m}(D)\right)=1$. In particular $M_{m}(D)$ has stable rank closed by extensions.

(3) Let

$$
0 \longrightarrow I \longrightarrow B \longrightarrow 0
$$

be a unital extension of $A$. If $\operatorname{er}(A)<n$ and $I$ has a local unit $\left(g_{i}\right)$ such that $\operatorname{sr}\left(g_{i} I g_{i}\right) \leq n$ and $\operatorname{er}\left(g_{i} I g_{i}\right)<n$ for all $i$, then $\operatorname{sr}(B)=n$ and $\operatorname{er}(B)<n$.

Proof. (1) This is essentially contained in [13. We include a sketch of the proof for the convenience of the reader. Assume that we have a unital extension $B$ of $A$ with $\operatorname{sr}(I) \leq n$. Let $\mathbf{a}=\left(a_{1}, \ldots, a_{n+1}\right)^{t} \in U_{c}(n+1, B)$. Then $\overline{\mathbf{a}}=$ $\left(\overline{a_{1}}, \ldots, \overline{a_{n+1}}\right)^{t} \in U_{c}(n+1, A)$. Since $\operatorname{sr}(A)=n$, there exist $b_{1}, \ldots, b_{n} \in B$ such that $\left(\overline{a_{1}}+\overline{b_{1}} \overline{a_{n+1}}, \ldots, \overline{a_{n}}+\overline{b_{n}} \overline{a_{n+1}}\right)^{t} \in U_{c}(n, A)$. Replacing a with $\left(a_{1}+b_{1} a_{n+1}, \ldots, a_{n}+\right.$ $\left.b_{n} a_{n+1}, a_{n+1}\right)$, we can assume that $\left(\overline{a_{1}}, \ldots, \overline{a_{n}}\right)^{t} \in U_{c}(n, A)$.

Since $\operatorname{er}(A) \leq n-1$, there exists $E \in E(n, B)$ such that $\bar{E} \cdot\left(\overline{a_{1}}, \ldots, \overline{a_{n}}\right)^{t}=$ $(1,0, \ldots, 0)^{t}$. Since $\mathbf{a}$ is reducible if and only if $\operatorname{diag}(E, 1) \cdot \mathbf{a}$ is reducible, we can assume that $\left(\overline{a_{1}}, \ldots, \overline{a_{n}}\right)^{t}=(1,0, \ldots, 0)^{t}$. Finally, replacing $a_{n+1}$ with $a_{n+1}-$ $a_{1} a_{n+1}$, we can assume that $\overline{\mathbf{a}}=(1,0, \ldots, 0)^{t}$, that is, $\mathbf{a} \in U_{c}(n+1, I)$. Now, as $\operatorname{sr}(I) \leq n$, a is reducible in $I$, and so in $B$, as desired.

Given any positive integer $m \geq 1, \operatorname{sr}\left(M_{m}(A)\right)=\lceil(\operatorname{sr}(A)-1) / m\rceil+1$ by $[13$, Theorem 3] and $\operatorname{er}\left(M_{m}(A)\right) \leq\lceil\operatorname{er}(A) / m\rceil$ by [10, Theorem 11.5.15]. So, it is clear that $\operatorname{er}(A)<\operatorname{sr}(A)$ implies $\operatorname{er}\left(M_{m}(A)\right)<\operatorname{sr}\left(M_{m}(A)\right)$. Hence, by the first part of the proof, $M_{m}(A)$ has stable rank closed by extensions, as desired.

(2) It is well known that a Euclidean domain has stable rank less than or equal to 2, and that it has elementary rank equal to 1; see e.g. [10, Proposition 11.5.3]. So, the result follows from part (1).

(3) Since $\operatorname{sr}(I) \leq n$, the fact that $\operatorname{sr}(B)=n$ follows from part (1). Now, take $m \geq n$, and set $\mathbf{a}=\left(a_{1}, \ldots, a_{m}\right)^{t} \in U_{c}(m, B)$. Since $\operatorname{er}(A)<n$, there exists $E \in E(m, B)$ such that $\bar{E} \cdot \overline{\mathbf{a}}=(1,0, \ldots, 0)^{t}$. So, $\mathbf{b}:=E \cdot \mathbf{a} \equiv(1,0, \ldots, 0)^{t}(\bmod I)$. Let $g \in I$ be an idempotent in the local unit such that $b_{1}-1, b_{2}, \ldots, b_{m} \in g I g$. Since $\operatorname{er}(g I g)<n$ by hypothesis, there exists $G \in E(m, g I g)$ such that $(G+\operatorname{diag}(1-g$, $\ldots, 1-g)) \cdot \mathbf{b}=(1,0, \ldots, 0)^{t}$.

Corollary 2.6. Let $A$ be a unital $K$-algebra with $\operatorname{sr}(A)=n \geq 2$ and $\operatorname{er}(A)<\operatorname{sr}(A)$. Then, for any not necessarily unital $K$-algebra $B$ and two-sided ideal $I$ of $B$ such that $B / I \cong A$ and $\operatorname{sr}(I) \leq n$, we have $\operatorname{sr}(B)=n$.

Proof. Given any $K$-algebra $R$, we define the unitization $R^{1}=R \times K$, with the product

$$
(r, a) \cdot(s, b)=(r s+a s+r b, a b) .
$$

Consider the unital extension

$$
0 \longrightarrow I \longrightarrow B^{1} \longrightarrow A^{1} \longrightarrow 0 .
$$


Notice that $A^{1} \cong A \times K$, because $A$ is unital. So, $\operatorname{sr}\left(A^{1}\right)=\operatorname{sr}(A)$ and $\operatorname{er}\left(A^{1}\right)=$ $\operatorname{er}(A)$. Now, by Lemma 2.5(1), $\operatorname{sr}\left(B^{1}\right) \leq n$. Since $n \leq \operatorname{sr}(B) \leq \operatorname{sr}\left(B^{1}\right) \leq n$, the result holds.

Proposition 2.7. Let $E$ be a finite graph with isolated cycles. Then $\operatorname{sr}(L(E)) \leq 2$ and $\operatorname{er}(L(E))=1$. Moreover, $\operatorname{sr}(L(E))=1$ if and only if $E$ is acyclic.

Proof. We proceed by induction on the number of cycles of $E$. If $E$ has no cycles, then $\operatorname{sr}(L(E))=1$ by Lemma 2.1, so that $\operatorname{er}(L(E))=1$ by [10, Proposition 11.3.11]. Assume that $E$ has cycles $C_{1}, \ldots, C_{n}$. Define a binary relation on the set of cycles by setting $C_{i} \geq C_{j}$ iff there exists a finite path $\alpha$ such that $s(\alpha) \in C_{i}^{0}$ and $r(\alpha) \in C_{j}^{0}$. Since $E$ is a graph with isolated cycles, $\geq$ turns out to be a partial order on the set of cycles. Since the set of cycles is finite, there exists a maximal one, say $C_{1}$. Set $A=\left\{e \in E^{1} \mid s(e) \in C_{1}\right.$ and $\left.r(e) \notin C_{1}\right\}$, let $S(E)$ denote the set of sinks of $E$, and define $B=\{r(e) \mid e \in A\} \cup S(E) \cup \bigcup_{i=2}^{n} C_{i}^{0}$. Let $H$ be the hereditary and saturated closure of $B$. By construction of $H, C_{1}$ is the unique cycle in $E / H$, and it has no exits. Moreover, $E / H$ coincides with the hereditary and saturated closure of $C_{1}$. Then, $L(E / H) \cong M_{k}\left(K\left[x, x^{-1}\right]\right)$ for some $k \geq 1$. Consider the extension

$$
0 \longrightarrow I(H) \longrightarrow L(E) \longrightarrow L(E / H) \longrightarrow 0 .
$$

Now, by Lemma 2.5 $(2), \operatorname{sr}(L(E / H))=2$ and $\operatorname{er}(L(E / H))=1$. Consider the local unit $\left(p_{X}\right)$ of $L\left({ }_{H} E\right) \cong I(H)$ consisting of idempotents $p_{X}=\sum_{v \in X} v$ where $X$ ranges over the set of vertices of ${ }_{H} E$ containing $H$. Since these sets are hereditary in $\left({ }_{H} E\right)^{0}$, we get that $p_{X} I(H) p_{X}=p_{X} L\left({ }_{H} E\right) p_{X}=L\left(\left({ }_{H} E\right)_{X}\right)$ is a path algebra of a graph with isolated cycles, containing exactly $n-1$ cycles. By the induction hypothesis, $\operatorname{sr}\left(p_{X} I(H) p_{X}\right) \leq 2$ and $\operatorname{er}\left(p_{X} I(H) p_{X}\right)=1$. So, by Lemma 2.5(3), we conclude that $\operatorname{sr}(L(E))=2$ and $\operatorname{er}(L(E))=1$. Hence, the induction step works, so we are done.

We are now ready to obtain our main result.

Theorem 2.8. Let $E$ be a row-finite graph. Then the values of the stable rank of $L(E)$ are:

(1) $\operatorname{sr}(L(E))=1$ if $E$ is acyclic;

(2) $\operatorname{sr}(L(E))=\infty$ if there exists $H \in \mathcal{H}_{E}$ such that the quotient graph $E / H$ is nonempty, finite, cofinal, contains no sinks and each cycle has an exit;

(3) $\operatorname{sr}(L(E))=2$ otherwise.

Proof. (1) derives from Lemma 2.1, while (2) derives from Lemma 2.2 and Lemma 1.6. We can thus assume that $E$ contains cycles and, using Lemma 1.6, that $L(E)$ does not have any unital purely infinite simple quotient.

By Lemma 2.3, there exists a hereditary saturated set $X$ of $E^{0}$ such that $\operatorname{sr}(I(X)) \leq 2$, while $E / X$ is a graph having isolated cycles. By [5, Lemma 3.1], there is an ascending sequence $\left(E_{i}\right)$ of complete finite subgraphs of $E / X$ such that $E / X=\bigcup_{i \geq 1} E_{i}$. So, by [5, Lemma 3.2], $L(E / X) \cong \lim _{\longrightarrow} L\left(E_{i}\right)$. For each $i \geq 1$, there is a natural graded $K$-algebra homomorphism $\phi_{i}: L\left(E_{i}\right) \rightarrow L(E / X)$. The kernel of $\phi_{i}$ is a graded ideal of $L\left(E_{i}\right)$ whose intersection with $E_{i}^{0}$ is empty, so $\phi_{i}$ is injective and the image $L_{i}$ of $L\left(E_{i}\right)$ through $\phi_{i}$ is isomorphic with $L\left(E_{i}\right)$. It follows from Proposition 2.7 that, for every $i \geq 1, \operatorname{sr}\left(L_{i}\right) \leq 2$ and $\operatorname{er}\left(L_{i}\right)=1$. If $\pi: L(E) \rightarrow L(E / X)$ denotes the natural epimorphism (see [7, Lemma 2.3(1)]), 
then given any $i \geq 1$, we have

$$
0 \longrightarrow I(X) \longrightarrow \pi^{-1}\left(L_{i}\right) \longrightarrow L_{i} \longrightarrow 0 \text {. }
$$

If $\operatorname{sr}\left(L_{i}\right)=1$, then $\operatorname{sr}\left(\pi^{-1}\left(L_{i}\right)\right) \leq 2$ by [13, Theorem 4]. If $\operatorname{sr}\left(L_{i}\right)=2$, then it follows from Corollary 2.6 that $\operatorname{sr}\left(\pi^{-1}\left(L_{i}\right)\right)=2$. Since $L(E)=\bigcup_{i>1} \pi^{-1}\left(L_{i}\right)$ we get that $\operatorname{sr}(L(E)) \leq 2$. Since $E$ contains cycles we have that either $I(X) \neq 0$ or $E / X$ contains cycles. If $I(X) \neq 0$, then $\operatorname{sr}(I(X))=2$ by Lemma 2.3 and so $\operatorname{sr}(L(E))=2$ by [13, Theorem 4]. If $I(X)=0$, then $E$ has isolated cycles. Take a vertex $v$ in a cycle $C$ of $E$ and let $H$ be the hereditary subset of $E$ generated by $v$. Observe that $L\left(E_{H}\right)=p L(E) p$ for the idempotent $p=\sum_{w \in H^{0}} w \in \mathcal{M}(L(E))$, where $\mathcal{M}(L(E))$ denotes the multiplier algebra of $L(E)$; see [6]. Let $I$ be the ideal of $p L(E) p$ generated by all the basic idempotents $r(e)$ where $e \in E^{1}$ is such that $s(e) \in C$ and $r(e) \notin C$. Since $E$ has isolated cycles it follows that $I$ is a proper ideal of $p L(E) p$ and moreover $p L(E) p / I \cong M_{k}\left(K\left[x, x^{-1}\right]\right)$, where $k$ is the number of vertices in $C$. We get

$$
\operatorname{sr}(p L(E) p) \geq \operatorname{sr}(p L(E) p / I)=2 .
$$

It follows that $1<\operatorname{sr}(L(E)) \leq 2$ and thus $\operatorname{sr}(L(E))=2$, as desired.

\section{Some REMARKS AND EXAMPles}

In this section we present several examples of Leavitt path algebras, and we compute their stable rank by using Theorem 2.8. Several remarks on the relationship with the stable rank of graph $C^{*}$-algebras are also included.

Examples 3.1. The basic examples to illustrate Theorem 2.8 coincide with those given in [1, Example 1.4]:

(1) The Leavitt path algebra associated with the acyclic graph $E$

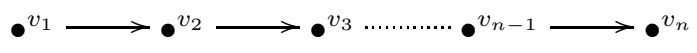

satisfies $L(E) \cong M_{n}(K)$. Thus, $\operatorname{sr}(L(E))=1$ by Theorem 2.8(1) (in this particular case, the original result is due to Bass).

(2) For $n \geq 2$, the Leavitt path algebra associated with the graph $F$

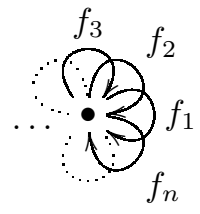

is an example of a unital purely infinite simple algebra, because of 2, Theorem 11]; in fact, $L(F) \cong L(1, n)$ (the $n$th Leavitt algebra) by [2, Example 12(ii)]. Thus, $\operatorname{sr}(L(F))=\infty$ by Theorem 2.8(2) (in this particular case, one can also trace this fact using [12, Proposition 6.5]).

(3) Finally, the Leavitt path algebra associated with the graph $G$

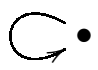

satisfies $L(G) \cong K\left[z, z^{-1}\right]$ by [1, Example 1.4(ii)]. Thus, $\operatorname{sr}(L(G))=2$ by Theorem 2.8(3).

Examples 3.2. We show some further examples that illustrate the complexity of the models of Leavitt path algebras: 
(1) On the one hand, stable rank 2 examples can be obtained as more or less complex extensions of the ring of Laurent polynomials, as we can see with the Leavitt path algebra of the graph $E$

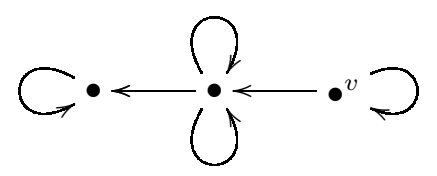

Here the ideal $I$ in Lemma2.3 is $I=I\left(E^{0} \backslash\{v\}\right)$, since $L(E) / I \cong K\left[x, x^{-1}\right]$. Notice that, because of Lemma 2.3 $\operatorname{sr}(I)=2$, while $\operatorname{sr}(L(E))=\operatorname{sr}(L(E) / I)$ $=2$ by Theorem 2.8(3). The remarkable fact behind Theorem 2.8 is that in the context of Leavitt path algebras, extensions of stable rank 2 rings by stable rank 2 ideals cannot attain stable rank 3 (in general this is not true).

(2) On the other hand, unital purely infinite simple Leavitt path algebras turn out to be more complex than the classical Leavitt algebras, so that there are plenty of unital Leavitt path algebras with infinite stable rank different from the classical examples. For example, the Leavitt path algebra of the graph $F$

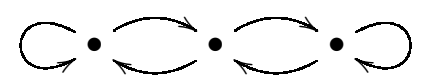

is unital purely infinite simple by [2, Theorem 11], but $\left(K_{0}(L(F)),[1]\right) \cong$ $(\mathbb{Z}, 0)$ by $\left[5\right.$, Theorem 3.5] and [4, Corollary 2.2], while $\left(K_{0}(L(1, n)),[1]\right) \cong$ $(\mathbb{Z} /(n-1) \mathbb{Z}, \overline{1})$ (see 4 , Theorem 4.2$])$. Taking the graph $G$

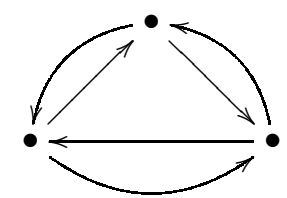

instead of $F$, we get a unital purely infinite simple Leavitt path algebra such that

$$
\left(K_{0}(L(G)),[1]\right) \cong((\mathbb{Z} / 2 \mathbb{Z}) \oplus(\mathbb{Z} / 2 \mathbb{Z}),(\overline{0}, \overline{0})) .
$$

An extra example is that associated with the graph $H$

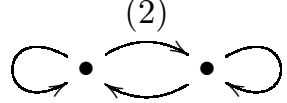

(4)

(here the $(n)$ s denote the number of parallel edges), which is again unital purely infinite simple, and such that

$$
\left(K_{0}(L(H)),[1]\right) \cong(\mathbb{Z} \oplus(\mathbb{Z} / 2 \mathbb{Z}),(1, \overline{1})) .
$$

Not one of them can be, then, isomorphic to any classical Leavitt algebra.

Remark 3.3. Fix $K=\mathbb{C}$, the field of complex numbers, and let $E$ be any row-finite graph. Then:

(1) It follows from [8, Proposition 3.1 and Theorem 3.4(2)] that $\operatorname{sr}\left(C^{*}(E)\right)=\infty$ if and only if there exists $H \in \mathcal{H}_{E}$ such that the quotient graph $E / H$ is nonempty, finite, cofinal, contains no sinks and each cycle has an exit. 
By using this and Theorem 2.8, we see that $\operatorname{sr}(L(E))=\infty$ if and only if $\operatorname{sr}\left(C^{*}(E)\right)=\infty$.

(2) Since any acyclic graph is a graph whose cycles have no exits, we have that $\operatorname{sr}(L(E))=1$ implies that $\operatorname{sr}\left(C^{*}(E)\right)=1$.

(3) So, the only difference occurs when $\operatorname{sr}(L(E))=2$ and all the cycles in $E$ have no exits, since then $\operatorname{sr}\left(C^{*}(E)\right)=1$ by [8, Theorem 3.4(1)]. The simplest example of this situation is the graph $G$ in Example 3.1(3). As we noticed, $L(G) \cong \mathbb{C}\left[z, z^{-1}\right]$ and $\operatorname{sr}(L(G))=2$. It is clear that

$$
(1+z) \mathbb{C}\left[z, z^{-1}\right]+\left(1+z^{2}\right) \mathbb{C}\left[z, z^{-1}\right]=\mathbb{C}\left[z, z^{-1}\right],
$$

and it is straightforward to see that there is no element $v \in \mathbb{C}\left[z, z^{-1}\right]$ such that $(1+z)+v\left(1+z^{2}\right)$ is invertible in $\mathbb{C}\left[z, z^{-1}\right]$. On the other hand, if we take the completion of $L(G)$, we get the graph $C^{*}$-algebra $C^{*}(G) \cong \mathcal{C}(\mathbb{T})$, which has stable rank 1 by [12, Proposition 1.7]. Because of [9], there exists $v \in C^{*}(E)$ such that $(1+z)+v\left(1+z^{2}\right)$ is invertible in $\mathcal{C}(\mathbb{T})$. Since a continuous function in $\mathcal{C}(\mathbb{T})$ is invertible if and only if it has no zeroes in $\mathbb{T}$, we see that we can take $v=1$.

Remark 3.4. Stable rank is not Morita invariant in general, but in the case of Leavitt path algebras some interesting phenomena arise:

(1) Suppose that $E, F$ are finite graphs such that $L(E)$ and $L(F)$ are Morita equivalent. Thus, $L(E) \cong P \cdot M_{n}(L(F)) \cdot P$ for some $n \in \mathbb{N}$ and some full idempotent $P \in M_{n}(L(F))$. Since the values 1 and $\infty$ in the stable rank are preserved by passing to matrices [13, Theorem 4] and full corners [3, Theorem 7 and Theorem 8], Theorem 2.8 implies that $\operatorname{sr}(L(E))=\operatorname{sr}(L(F))$. So, stable rank is a Morita invariant for unital Leavitt path algebras.

(2) This is no longer true when $L(E)$ is nonunital. To see an example, let $F$ be the graph in Example $3.1(2)$, and $F^{\infty}$ be the rose of $n$ petals

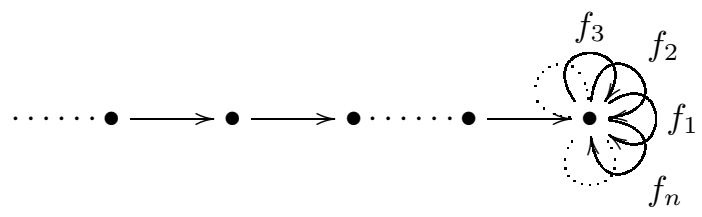

with an infinite tail added. As we have seen before, $L(F) \cong L(1, n)$ (the $n$th Leavitt algebra) with $\operatorname{sr}(L(F))=\infty$, while an easy induction argument using [2, Proposition 13] shows that $L\left(F^{\infty}\right) \cong M_{\infty}(L(1, n))$. Hence these two algebras are Morita equivalent. On the other hand, $L\left(F^{\infty}\right)$ has no unital purely infinite simple quotients (as it is simple and nonunital), so that $\operatorname{sr}\left(L\left(F^{\infty}\right)\right)=2$ by Theorem $2.8(3)$.

Moreover, the graph $F^{\infty}$ is a direct limit (see [5, Section 3]) of the graphs $E_{n}^{m}$

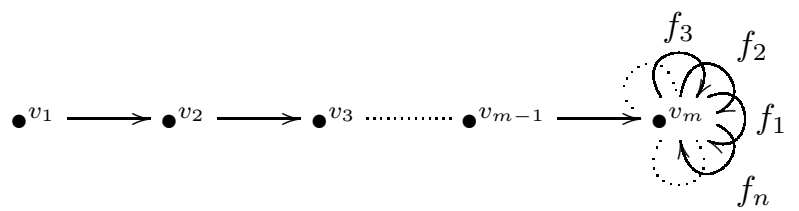


introduced in [2, Example 12]. Since $L\left(F^{\infty}\right) \cong \lim _{\longrightarrow} L\left(E_{n}^{m}\right)$ and $L\left(E_{n}^{m}\right) \cong$ $M_{m}(L(1, n))$, we get $\operatorname{sr}\left(L\left(E_{n}^{m}\right)\right)=\infty$ by the above remark, whence

$$
2=\operatorname{sr}\left(L\left(F^{\infty}\right)\right)=\operatorname{sr}\left(\lim _{\longrightarrow} L\left(E_{n}^{m}\right)\right)<\liminf \operatorname{sr}\left(L\left(E_{n}^{m}\right)\right)=\infty .
$$

So, this inequality can be strict when we work with Leavitt path algebras.

\section{ACKNOWLEDGMENTS}

Part of this work was done during visits of the first author to the Departamento de Matemáticas de la Universidad de Cádiz (Spain) and of the second author to the Centre de Recerca Matemàtica (U.A.B., Spain). Both authors want to thank the host centers for their warm hospitality. Also they wish to thank the referee for his/her comments and suggestions.

\section{REFERENCES}

[1] G. Abrams, G. Aranda Pino, The Leavitt path algebra of a graph, J. Algebra 293 (2005), 319-334. MR2172342 (2007b:46085)

[2] G. Abrams, G. Aranda Pino, Purely infinite simple Leavitt path algebras, J. Pure Appl. Algebra 207 (2006), 553-563. MR2265539 (2007f:16062)

[3] P. Ara, K.R. Goodearl, Stable rank of corner rings, Proc. Amer. Math. Soc. 133(2) (2005), 379-386. MR2093058 (2005h:19001)

[4] P. Ara, K.R. Goodearl, E. Pardo, $K_{0}$ of purely infinite simple regular rings, $K$-Theory 26 (2002), 69-100. MR1918211 (2004e:19001)

[5] P. Ara, M.A. Moreno, E. Pardo, Nonstable K-theory for graph algebras, Algebra Rep. Th. 10 (2007), 157-178. MR2310414

[6] P. Ara, F. Perera, Multipliers of von Neumann regular rings, Comm. in Algebra 28(7) (2000), 3359-3385. MR 1765323(2001i:16020)

[7] G. Aranda Pino, E. Pardo, M. Siles Molina, Exchange Leavitt path algebras and stable rank, J. Algebra 305 (2006), 912-936. MR2266860 (2007i:16009)

[8] K. Deicke, J.H. Hong, W. Szymański, Stable rank of graph algebras. Type I graph algebras and their limits, Indiana Univ. Math. J. 52(4) (2003), 963-979. MR2001940 (2004e:46065)

[9] R. H. Herman, L. N. Vaserstein, The stable range of $C^{*}$-algebras, Invent. Math. 77 (1984), 553-555. MR759256 (86a:46074)

[10] J. C. McConnell, J.C. Robson, "Noncommutative Noetherian Rings", John Wiley \& Sons, Chichester, 1987. MR934572 (89j:16023)

[11] I. Raeburn, "Graph Algebras", CBMS Reg. Conf. Ser. Math., vol. 103, Amer. Math. Soc., Providence, RI, 2005. MR2135030 (2005k:46141)

[12] M.A. Rieffel, Dimension and stable rank in the $K$-theory of $C^{*}$-algebras, Proc. London Math. Soc. 46(2) (1983), 301-333. MR693043 (84g:46085)

[13] L.N. VASERstein, The stable range of rings and the dimension of topological spaces, Func. Anal. Appl. 5 (1971), 102-110. MR0284476 (44:1701)

Departament de Matemàtiques, Universitat Autònoma de Barcelona, 08193 BellaTERra (BARCELONA), SPAin

E-mail address: para@mat.uab.cat

Departamento de Matemáticas, Universidad de Cádiz, Apartado 40, 11510 Puerto ReAl (CÁdiz), Spain

E-mail address: enrique.pardo@uca.es

URL: http://www2.uca.es/dept/matematicas/PPersonales/PardoEspino/index.HTML 